\title{
Acquisition of selective antitumoral effects of recombinant adeno-associated virus by genetically inserting tumor-targeting peptides into capsid proteins
}

\author{
HAN SAEM LEE ${ }^{1}$, JI YUN KIM ${ }^{1}$, WON IL LEE ${ }^{1}$, SUNG JIN KIM $^{1}$, MIN JI KO ${ }^{1}$, \\ SUNJOO JEONG ${ }^{4}$, KEERANG PARK ${ }^{5}, \mathrm{HAN} \mathrm{CHOE}^{2,3}$ and HEUIRAN LEE ${ }^{1,3}$
}

\begin{abstract}
Departments of ${ }^{1}$ Microbiology and ${ }^{2}$ Physiology, and ${ }^{3}$ Bio-Medical Institute of Technology, University of Ulsan College of Medicine, Seoul; ${ }^{4}$ Department of Molecular Biology, College of Natural Sciences, Dankook University, Geong-Gi; ${ }^{5}$ Department of Biotechnology, Juseong University, Chung-Buk, Republic of Korea
\end{abstract}

Received March 31, 2011; Accepted July 25, 2011

DOI: $10.3892 / 01.2011 .376$

\begin{abstract}
Recombinant adeno-associated virus serotype 5 (rAAV5) is considered to be a promising gene transfer vehicle. However, preferential gene delivery to the tumor remains a requirement for cancer treatment. We generated rAAV5 mutants bearing tumor marker-binding peptides and analyzed their properties as viral vectors, as well as their transduction efficiencies and preferential antitumoral potencies. All of the mutants were successfully produced. Transduction analyses showed that rAAV5 mutants harboring tumor-homing peptides, including RGD and $\mathrm{TnC}$, transduced human cancer cells expressing corresponding receptors on their surfaces. RGDS peptides and $\mathrm{TnC}$ antibodies significantly suppressed transduction by rAAV5-RGD and rAAV5-TnC. Cytotoxicity was evident upon transfer of HSV-TK to cells by re-targeted rAAV5. These results provide evidence that rAAV5 vectors, genetically armed with tumor-targeting ligands, preferentially infect human cancer cells harboring the corresponding receptors, thereby inducing antitumoral effects. Further optimization of rAAV5 mutant viruses should thus facilitate practical exploitation of these vectors for gene-based cancer treatment.
\end{abstract}

\section{Introduction}

Recombinant adeno-associated virus (rAAV), ehxibiting minimal toxicity and long-term gene expression, is a promising cancer gene therapy vector $(1,2)$. In particular, various studies have validated the potential of rAAV-secreting therapeutic gene products as a gene transfer tool to treat metastatic cancers

Correspondence to: Professor Heuiran Lee, Department of Microbiology, University of Ulsan College of Medicine, 86 Asanbyeongwon-gil Songpa-gu, 138-736, Seoul, Republic of Korea E-mail: heuiran@amc.seoul.kr

Key words: cancer gene therapy, recombinant adeno-associated virus serotype 5 , virus tropism, capsid modification, integrin, tenascin $\mathrm{C}$
$(3,4)$. Among these rAAV serotypes undergoing development as gene transfer tools, the rAAV2 vector has been analyzed most extensively, and is currently employed in clinical gene therapy $(3,5)$. Recently, we and other groups demonstrated the potential of the rAAV5 serotype as an effective vector for antitumoral gene delivery $(6,7)$. Other studies reported the lowest prevalence of AAV5-neutralizing antibodies and the highest prevalence of AAV2-neutralizing antibodies in the normal human population $(8,9)$. These findings thus support the hypothesis that rAAV5 vectors are beneficial in targeting therapeutic genes to tumors without the barrier of humoral immunity in humans.

However, rAAV5-mediated cancer gene therapy has certain limitations in relation to systemic administration in vivo. One of the major drawbacks of rAAV5-mediated gene delivery is the broad tissue tropism of rAAV5, which is not exclusively limited to tumor tissue. Transgene expression via rAAV5 is observed in tissues including the liver and spleen, unless vectors are administered directly to the target tissue or organ $(10,11)$. These data suggest that it is crucial to further improve the transduction properties of rAAV5 in order to facilitate the preferential delivery of transgenes to target tissues.

Consequently, the native tropism of rAAV5 was modified by genetically inserting various tumor-targeting peptide sequences into the capsid. The primary determinant of cell/ tissue tropism of rAAV is the binding specificity of the viral capsid to cellular receptors (12). Tumor-targeting motifs significantly enhance viral binding affinity to cell surface proteins, including integrins, sialyl Lewis X (sLeX), and tenascin C $(\mathrm{TnC})$, which are preferentially expressed in tumors (13-16). Since the initial attempt by Girod et al to deliver genes via mutated rAAV2 to cells resistant to rAAV2 infection (17), a number of investigators have successfully achieved re-targeted gene delivery, both in vivo and in vitro, by modifying the rAAV2 capsid through insertional mutagenesis (18-20). Currently, a limited number of studies focusing on re-targeted rAAV5 vectors are available. Thus, the aim of the present study was to identify the protruding loop regions capable of bearing tumortargeting motifs by modeling AAV5 capsid protein structures using a computational approach with the X-ray crystal 
Table I. Primer information used to construct plasmids containing genes of mutant rAAV5 capsids.

\begin{tabular}{|c|c|c|}
\hline \multirow{2}{*}{$\begin{array}{l}\text { Homing peptides } \\
\text { sLeX1 }\end{array}$} & \multicolumn{2}{|r|}{ Sequences of oligonucleotides ( 5 ' to $3^{\prime}$ ) } \\
\hline & Forward & TACAGCTCCCCCGCCACCACTGGCGGAGTCCAGTTCAAC \\
\hline & Reverse & TCTGGGGATCCAGTGGGCGTTATTTGTGCTCACGAAGCG \\
\hline \multirow[t]{2}{*}{ sLeX2 } & Forward & AGCTCCCCCGCCACCGGAGGCACTGCCCCCGCGACCGGCACGTACAACC \\
\hline & Reverse & GTATCTGGGGATCCAGTGGGCTCCGGTGGAGCTCTGGTTGTTGGTGGC \\
\hline \multirow[t]{2}{*}{ RGD1 } & Forward & CGACAGTCACAGCCGGTGGAGCTCTGGTTGTT \\
\hline & Reverse & AGGCGACTGTTTCTGTACTGCCCCCGCGACC \\
\hline \multirow[t]{2}{*}{ RGD2 } & Forward & TCCCTCGACAGTCACAGCCGGTGGAGCTCTGGTTGTT \\
\hline & Reverse & CTGTTTCTGTGGCCTGTCAACTGCCCCCGCGACC \\
\hline \multirow[t]{2}{*}{$\mathrm{TnC} 1$} & Forward & ATGAAAGCCGCAATCGCAGCCGGTGGAGCTCTGGTT \\
\hline & Reverse & AAACATAAAAGCTGCTTTTGCACTGCCCCCGCGACC \\
\hline \multirow[t]{2}{*}{$\mathrm{TnC} 2$} & Forward & TATGAAAGCCGGTGGAGCTCTGGTT \\
\hline & Reverse & AACATAAAAGCACTGCCCCCGCGACC \\
\hline \multirow[t]{2}{*}{$\mathrm{TnC} 3$} & Forward & CAGCGCCGGGCTTTTATGTTTATG \\
\hline & Reverse & AGCCCGGTGTGCTTTTGCACTGCC \\
\hline \multirow[t]{2}{*}{$\mathrm{TnC} 4$} & Forward & CTTTTATGTTTATGAAAGCCGGTGGAGCTCTGGTT \\
\hline & Reverse & CCCGGCGCTGAGCCCGGTGACTGCCCCCGCGACC \\
\hline
\end{tabular}

structure of AAV2 (21). Hypothetical atomic structures of insertion mutants at the expected loop region were constructed. Using this approach, various rAAV5 mutants with altered tropism were generated, which preferentially transduced tumors and subsequently induced antitumoral effects.

\section{Materials and methods}

Cell culture, virus preparation and transduction by rAAV vectors. $293 \mathrm{~T}$ and human cancer cells (hepatocellular SK-Hep1; cervical HeLa; breast MDA-MB-231 and MDA-MD-435S; glioblastoma U-251, U87-MG, U118-MG and U-251; and colon HCT-116) were purchased from the American Type Culture Collection (ATCC; Manassas, VA, USA). Cancer cells were maintained in Dulbecco's modified Eagle's medium supplemented with $10 \%$ fetal bovine serum, L-glutamine $(2 \mathrm{mM})$, penicillin $(100 \mathrm{IU} / \mathrm{ml})$ and streptomycin $(50 \mu \mathrm{g} / \mathrm{ml})$. The CHO-Lec2 cell line purchased from ATCC was maintained in $\alpha$-minimum essential medium supplemented with $10 \%$ fetal bovine serum, penicillin $(100 \mathrm{IU} / \mathrm{ml})$ and streptomycin $(50 \mu \mathrm{g} / \mathrm{ml})$.

Table I shows the primers used to construct plasmids containing genes of mutant rAAV5 capsids. Using pSp72 plasmid containing the Rep gene of AAV2 and the Cap gene of AAV5 as an original backbone, final constructs were generated by a series of cloning steps and sequencing conformation. Self-complementary $\mathrm{rAAV}$ vectors were generated as described elsewhere (6). Titration of rAAVs prepared using $\mathrm{CsCl}_{2}$ gradient ultracentrifugation was performed using TaqMan-based Q-PCRs (iQTM Supermix, Bio-Rad, Hercules, CA, USA).

Cells were infected with various rAAVs, as previously described (6). To maximize the transduction of rAAV vectors, adenovirus 5 was occasionally added. Transduction efficiencies (TEs) and means of fluorescence intensities (MFIs) were determined using a flow cytometer (FACSCalibur,
Becton-Dickinson, San Jose, CA, USA) and the CellQuest Plus program.

Computer modeling of capsid protein and $\mathrm{rAAV}$. Homology models of the capsid structures of rAAV5-sLeX1, rAAV5sLeX2 and rAAV5-RGD1 with a RGD-4C insert were generated on the basis of the crystal structure of AAV2 capsid (PDB ID code: 1LP3) using the homology modeling program Modeller 9v2 (22). The whole virion from a single capsid structure model was generated using the CNSsolve program v1.1 (23). Structural figures were prepared using PyMol v0.98 (www.pymol.org).

Cell surface marker analysis. Cells were detected with primary antibodies for $\alpha \mathrm{V} \beta 3 \mathrm{~b}$ and $\alpha \mathrm{V} \beta 5$ conjugated with FITC (LM609 and P1F6 clones: Chemicon, Temecula, CA, USA). To measure $\alpha 2,3$-sialic acids on the cell surface, cells were stained with primary MAL II lectin conjugated with biotin (Vector Laboratories, Burlingame, CA, USA), followed by the addition of secondary streptavidin-FITC. The proportion of immune-stained cells and the MFI of cells was measured using a flow cytometer.

RGD peptide and anti-tenascin C antibody competition assays. For the RGD peptide competition assay, cells in 24-well plates were pre-incubated in fresh medium supplemented with 200, $100,50,20$ or $10 \mathrm{mM}$ active RGDS or RGES peptide (Sigma, St. Louis, MO, USA) in the presence of $2 \%$ FBS for $30 \mathrm{~min}$ at room temperature. Then, rAAV5 (HeLa, MOI 10; U87-MG, 20; and SK-Hep1, 100) or rAAV5-RGD1 (HeLa, MOI 5x103; U87-MG, $5 \times 10^{3}$; and SK-Hep1, $1 \times 10^{4}$ ) mutants expressing GFP were added in the presence of peptides and adenovirus type 5 for $4 \mathrm{~h}$. For the anti-tenascin $\mathrm{C}$ antibody competition assay, cells in 96-well plates were pre-incubated in fresh medium supplemented with $1 \mathrm{mg} / \mathrm{ml}$ anti-tenascin C $\operatorname{IgG}(\mathrm{R} \& \mathrm{D}$ 
Table II. A summary of the general properties of the various rAAV5 mutants.

\begin{tabular}{llclc}
\hline Tumor markers & Viruses & Sites & \multicolumn{1}{c}{ Tumor-targeting peptides } & Titer (VG/ml) \\
\hline \multirow{3}{*}{ sLeX } & WT & - & - & $1.3 \times 10^{11}$ \\
\multirow{2}{*}{ Integrins } & sLeX1 & 444 & AHWIPRYSSPAT & $0.7 \times 10^{11}$ \\
& sLeX2 & 578 & G-AHWIPRYSSPAT-GG & $1.0 \times 10^{11}$ \\
TnC & RGD1 & 578 & G-CDCRGDCFC & $1.2 \times 10^{11}$ \\
& RGD2 & 578 & G-CDCRGDCFC-GLS & $1.0 \times 10^{11}$ \\
& TnC1 & 578 & GCDCG-FHKHK-SCFC & $1.1 \times 10^{11}$ \\
& TnC2 & 578 & G-FHKHKS & $1.9 \times 10^{11}$ \\
& TnC3 & 578 & GCDCG-FHKHKSPALSPV-CFC & $2.4 \times 10^{11}$ \\
& TnC4 & 578 & G-FHKHKSPALSPV & $2.0 \times 10^{11}$ \\
\hline
\end{tabular}

Systems, MAB2138, Minneapolis, AT, USA) (1:50, 1:20, and 1:10 dilution) with $2 \% \mathrm{FBS}$ for $30 \mathrm{~min}$ at room temperature. rAAV5 or rAAV5-TnC4 vectors containing a GFP-expressing cassette (virus titers at $20-50 \%$ transduction) were added in the presence of antibodies. After 2 days, TEs were measured. At least three independent competition assays were performed. Relative TEs were determined using the formula: (TE of rAAV with peptides/TE of rAAV only) x $100 \%$.

Cell cytotoxicity assay. U87-MG cells were seeded in a 6 -well plate. For $100 \%$ transgene transfer, cells were infected with rAAV5, rAAV5-RGD1 and rAAV5-TnC4 at MOIs of 200, 20,000 and 50,000, respectively. For 20\% transduction, 5-fold lower MOIs were employed. The following day, cells were infected with rAAV5 vectors expressing sc39TK for $24 \mathrm{~h}$. Cells $\left(2 \times 10^{3} /\right.$ well $)$ seeded in a 96-well plate were treated with various concentrations of the prodrug ganciclovir (Cymevene ${ }^{\circledR}$, Roche, Basel, Switzerland) for 5 days. Relative cell viabilities were determined as described previously (24).

Statistical analysis. Data were compared between groups using the Student's unpaired t-test, and values were considered significant at $\mathrm{p}<0.05$.

\section{Results}

Successful generation of rAAV5 vectors bearing genetically modified capsids. Alignment results with VP1 sequences of AAV2 and 5 previously obtained AAV2s $(17,25)$ indicate that peptides may be incorporated at positions 444 and 578 of rAAV5 VP1 (data not shown). Using eight different rep/cap plasmids encoding mutant rAAV5, capsid proteins bearing the eight peptide sequences (Table II) were generated. Analysis of the packaging ability of the rAAV5 mutants revealed that mutant virus production was generally as efficient as that of wild-type rAAV5. The buoyant densities of mutants within a narrow range of 1.370 to $1.385 \mathrm{~g} / \mathrm{ml}$ of the $\mathrm{CsCl}_{2}$ gradient were similar to that of the wild-type virus $(1.394 \pm 0.007 \mathrm{~g} / \mathrm{ml})$. Moreover, transmission electron microscopy data confirmed that the mutant vectors maintained structural integrity (data not shown). These findings suggest the possibility of insertional modification of rAAV5 at positions 444 and 578 in VP1 without disrupting the general conformation. $r A A V 5$ containing the RGD motif transduces a variety of human cancer cells in an integrin-dependent manner. Various cancer cells expressing $\alpha \mathrm{V} \beta 3$ or $\alpha \mathrm{V} \beta 5$ integrins were transduced with rAAV5-RGD1 or rAAV5 expressing GFP. rAAV5-RGD1 efficiently transduced integrin-positive cells (HeLa, U87-MG; Fig. 1A, MDA-MB-435S, and SK-Hep1; data not shown), but not integrin-negative MDA-MB-231 cells. Similar patterns of gene transfer were observed for rAAV5-RGD2 (data not shown). In contrast, rAAV5 transduced all cells expressing sialic acid, a major receptor, regardless of the degree of integrin expression. The $\alpha \mathrm{V} \beta 5$ integrin-positive and sialic acid-negative CHO-Lec 2 cells were transduced by rAAV5RGD1, but not rAAV5 (Fig. 1B).

Gene transfer by rAAV5-RGD1 in integrin-positive cells was significantly inhibited by the RGDS peptide in a concentration-dependent manner, but not by the non-specific RGES peptide (Fig. 1C). As expected, the TE of the rAAV5 virus was not disrupted by either peptide. These results collectively indicate that rAAV5 bearing the RGD motif preferentially transduces human cancer cells expressing integrins, regardless of the presence of sialic acid, the native receptor of the wild-type virus.

rAAV5-TnC variants preferentially transduce TnC-expressing cells. The TEs of rAAV5-TnC mutants measured in TnCpositive or -negative human cancer cells were dependent on $\mathrm{TnC}$ expression. rAAV5-TnC4 efficiently transduced TnC-positive U87-MG, but not TnC-negative HCT-116 (Fig. 2A). The antiTnC antibody specifically decreased the transduction level of rAAV5-TnC4 (Fig. 2B). The TEs of rAAV5-TnC4 in U87-MG cells decreased to $13.9 \pm 10.1$ and $40.9 \pm 12.0 \%$ at $1: 50$ and 1:20 antibody dilutions, respectively, compared to those of the mock-treated group. In contrast, TEs of rAAV5 were not altered in the presence of anti-TnC antibody. The data indicate that rAAV5-TnC specifically infects human cancer cells in a TnC-dependent manner. In conjunction with data from Fig. 2, the results suggest that the newly inserted peptides into the 578 amino acid position of rAAV5 VP1 facilitate recognition of their corresponding surface receptors and enhance virus targeting.

Genetically re-targeted rAAV5s expressing sc39TK induces antitumoral effects. To assess the therapeutic effects of 
A
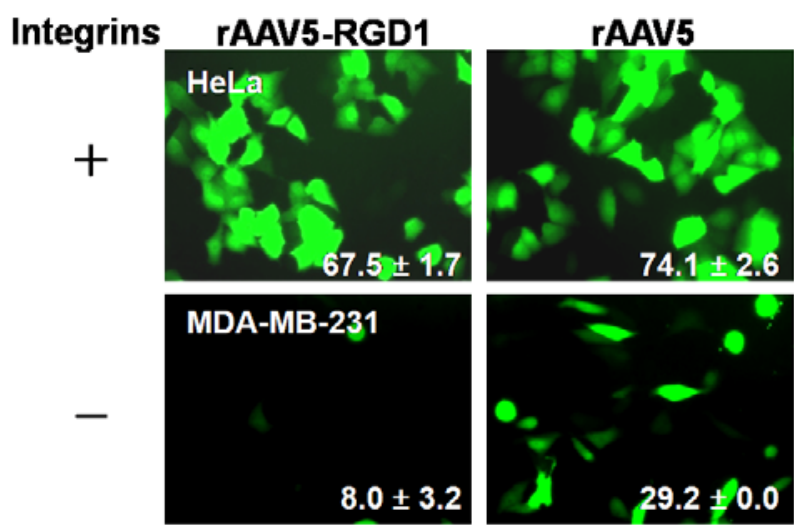

C

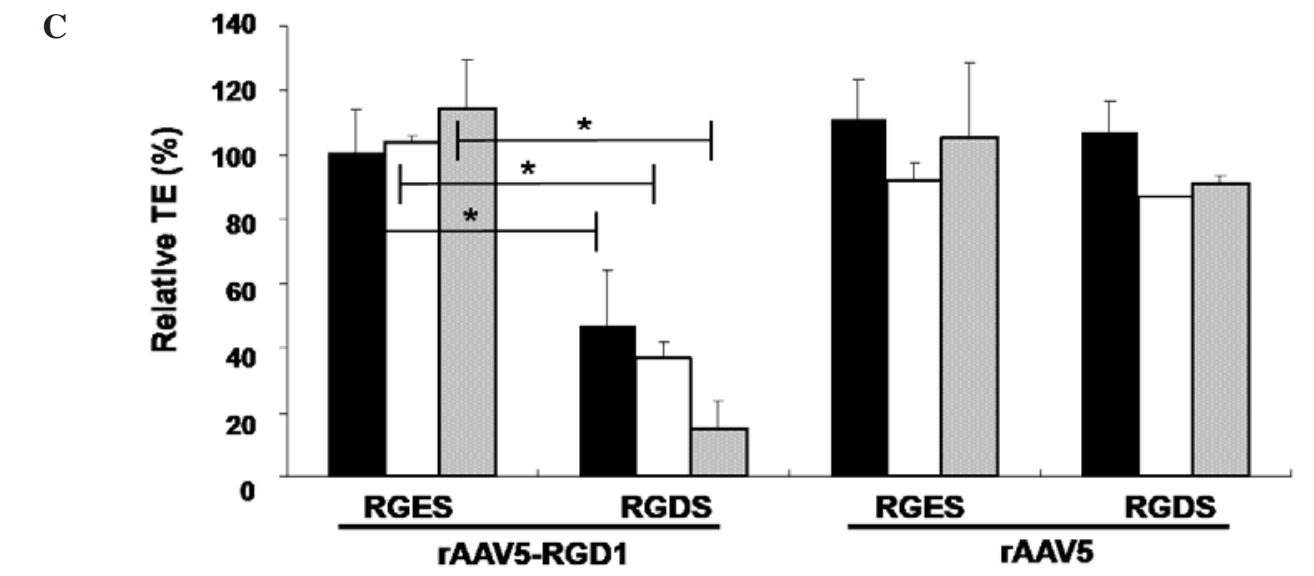

B

rAAV5-RGD1

\section{CHO-Lec2}

$\mathbf{p}$

$28.5 \pm 5.4$

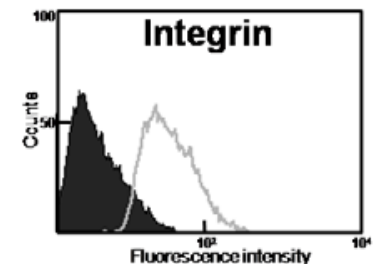

$4.8 \pm 3.0$

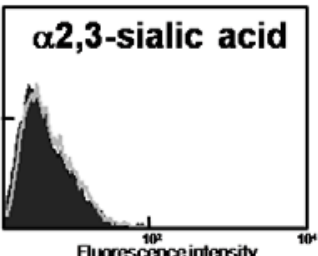

rAAV5

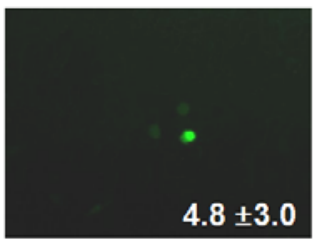

Fuorescence intensity

Figure 1. Integrin-dependent transduction of human cancer cells by rAAV5-RGD1. (A) Integrin-positive cells were infected with rAAV5-RGD1 (MOI 5,000) or rAAV5 (MOI 20) expressing GFP. After $48 \mathrm{~h}$, transduction efficiencies (TEs) were analyzed using a flow cytometer, as indicated in each image (mean \pm SD, $\mathrm{n} \geq 3$ ). (B) Similarly, CHO-Lec2 cells were treated with rAAV5-RGD1 (MOI 10,000) or rAAV5 (MOI 1,000). Flow cytometry was performed after incubating cells with either integrin antibody or lectin (n=3). (C) U87-MG cells were infected with rAAV5 or rAAV5-RGD1 in the absence/presence of an integrin-binding motif, RGDS peptide (black bars, $50 \mu \mathrm{M}$; white bars, $100 \mu \mathrm{M}$; gray bars, $200 \mu \mathrm{M}$ ), and the relative TE was determined. The non-specific RGES peptide was employed as a control. rAAV5-RGD1 efficiently transduced cells expressing integrins ( $\left.\mathrm{n}=3,{ }^{*} \mathrm{p}<0.005\right)$.

A
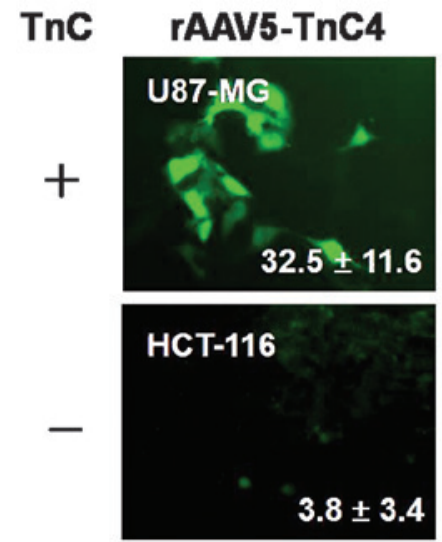

rAAV5
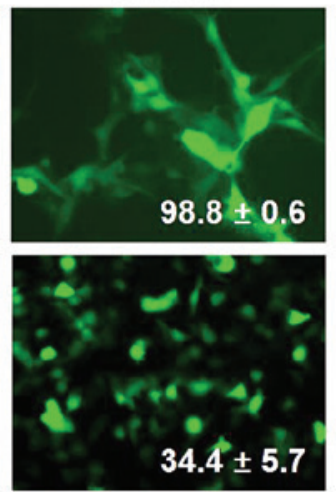

B

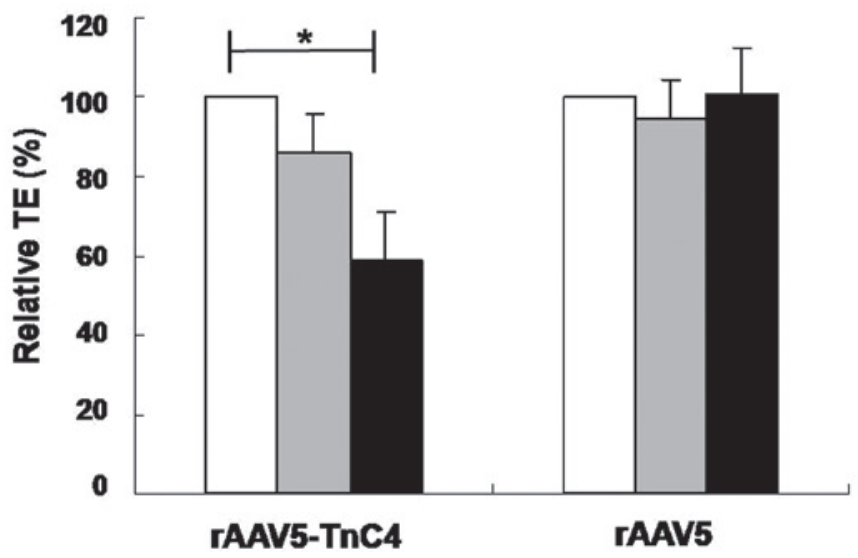

Figure 2. Tenascin C (TnC)-mediated transduction of rAAV5-TnC4 in human cancer cells. Cells were infected with rAAV5 (MOI 100) or rAAV5-TnC4 (MOI 20,000) in the presence/absence of TnC antibody, and the TEs were determined. (A) Efficient transduction of TnC-positive U87-MG cells by rAAV5-TnC4, and not TnC-negative HCT-116 cells is shown. (B) The degree of anti-TnC IgG-mediated blocking of rAAV5-TnC4 transduction in U87-MG cells (n=3, *p<0.005) is shown (white bars, no antibody; gray bars, 1:50 dilution; black bars, 1:20 dilution).

re-targeted rAAV5, we utilized the herpes simplex type thymidine kinase (HSV-sc39TK) gene/prodrug ganciclovir (GCV) system. sc39TK is a splicing-corrected version of
sr39TK, a mutant HSV-TK with increased affinity for GCV or acyclovir $(26,27)$. The sc39TK gene was delivered to U87-MG cells via rAAV5-RGD1, rAAV5-TnC4 and rAAV5 (Fig. 3). 


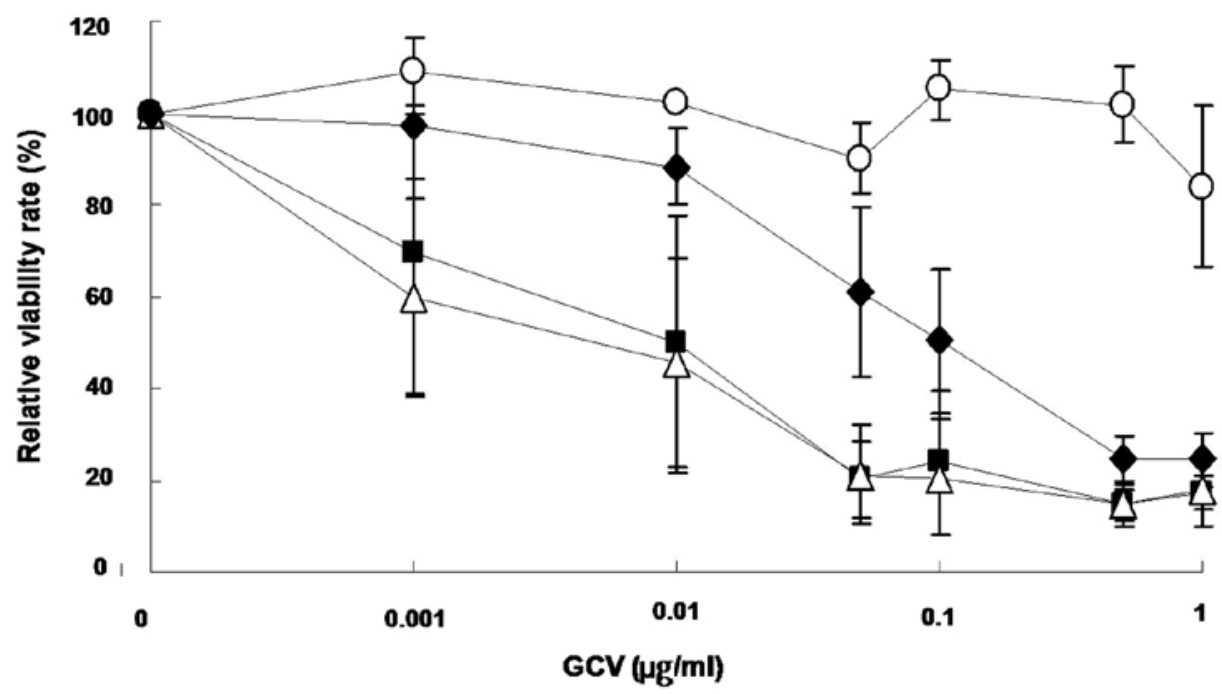

Figure 3. Evaluation of cytotoxicity upon expression of sc39TK genes and GCV treatment. U87-MG cells were infected with rAAV5, rAAV5-RGD1, rAAV5TnC4-expressing sc39TK, or rAAV5-GFP as a control. At one day after infection, the cells were incubated with GCV at the designated concentrations for another 5 days, and the relative cell viability was determined $(n=3)$. The cells transduced with sc39TK induced cytotoxicity in the presence of GCV in a

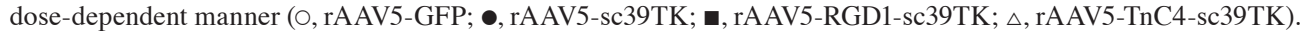

A

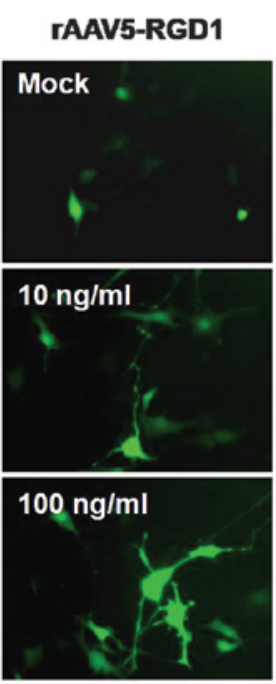

rAAV5-TnC4

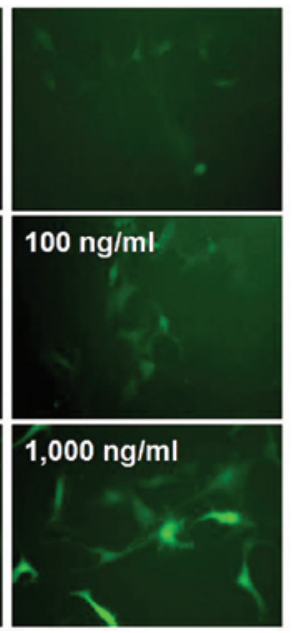

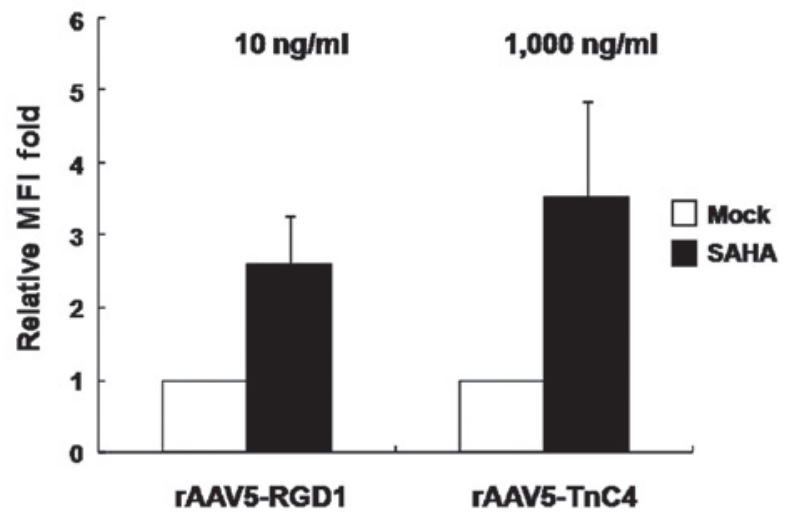

Figure 4. Effect of SAHA on transduction by rAAV5-RGD1 or rAAV5-TnC4. U87-MG cells under 10 to 1,000 ng/ml SAHA treatment, were infected with either rAAV5-RGD1 (MOI 500) or -TnC4 (MOI 10,000). (A) The degree of GFP expression is shown (magnification, x200). (B) The effect of SAHA on mean fluorescence intensities (MFIs) is shown. Cells were infected with rAAV5-RGD1 or rAAV5-TnC4 in the presence of $10 \mathrm{and} 1,000 \mathrm{ng} / \mathrm{ml} \mathrm{SAHA,} \mathrm{respectively.}$ MFIs of rAAV5 mutants were significantly increased under SAHA treatment conditions $(n=3)$.

The sc39TK gene transfer induced selective cytotoxicity in the presence of GCV, while GFP did not. Thus, it appears that re-targeted antitumoral gene transfer by mutant rAAV5s induces cytotoxicity in transduced tumor cells. Moreover, the degree of antitumoral effects differs with various rAAV5 mutants, possibly due to differences in gene transfer efficacy.

The HDAC inhibitor enhances gene delivery efficacy of re-targeted rAAV5 vectors. To further enhance rAAV5-RGD1 or-TnC4-mediated gene expression in target cancer cells, various concentrations of the antitumoral HDAC inhibitor, suberoylanilide hydroxamic acid (SAHA), were added prior to transduction with rAAV5-RGD1 and -TnC4. Enhanced GFP gene expression was observed with the two rAAV5 mutants in a dose-dependent manner following SAHA treatment (Fig. 4A). Additionally, SAHA enhanced the intensity of GFP expression per cell, as noted in the MFI values (Fig. 4B). In other words, a similar degree of gene expression may be achieved at 10 times lower the total particle concentrations if SAHA is introduced. Therefore, a combination of SAHA treatment and tumor-targeted rAAV5 vectors encoding antitumoral genes may significantly enhance therapeutic effects.

\section{Discussion}

For genetic re-targeting of rAAV against its natural tropism, rAAV5 has considerable potential as an attractive backbone due to favorable characteristics, such as cancer tropism, a low 
level of neutralizing antibodies in human populations, and superior virus production yield over other serotypes $(6,9,28)$. Limited information is available on rAA5 modification at present (29), although several studies have focused on genetic modification of rAAV1 and 2 capsids $(20,30,31)$. In the present study, it was demonstrated that i) rAAV5 vectors may be genetically modified within the capsid region, particularly at VP1 positions 444 and 578 ,ii) mutants bearing insertion sequences within a certain size limit are efficiently produced with no loss of viral properties, iii) the insertion of tumor-binding motifs in the rAAV5 capsid protein suppresses the native tropism of the rAAV5 vector, and iv) tumor-targeted rAAV5 vectors are capable of selectively transducing human cancer cells, depending on their own target receptor properties.

$\alpha \mathrm{V}$ integrin, sLeX and TnC are often over-expressed on the surfaces of a wide variety of tumor cells (14-16). Homing peptides of various sizes ranging from 7 to 20 amino acids were successfully inserted, which included sequences responding to tumor surface markers. Introduction of these peptides did not alter the physical or biological properties of viruses. The data suggest that rAAV5 tolerates up to 20 amino acids in terms of insertion size. Previously, Grifman et al performed an alignment of the AAV1-5 capsid sequences, and demonstrated that loops III and IV including Arg at positions 447 and 587 of rAAV2, respectively, could be freely modified to alter rAAV2 tropism without losing virus replication ability (18). Consistent with earlier findings, the two loops containing amino acids 444 and 578 of rAAV5 were genetically altered with no effects on rAAV5 production ability.

rAAV5 mutants harboring peptide sequences with affinities for specific tumor markers facilitate recognition of the corresponding cell surface receptors, and not the native sialic acid receptor. Along with systemic mutation analyses (25), the atomic structure of AAV2 strongly suggests that the primary receptor binding motif is located in the IV loop at position 587 near the 3-fold proximal peak. Another loop III, responsible for receptor recognition, resides on the distal floor of the peak. Walters et $\mathrm{al}$ suggested that three amino acids (I528, N546 and M547) located in the depression at the 2-fold axis are involved in the receptor binding of rAAV5 (32). However, we observed significant decreases in TE through the introduction of homing peptides at positions 444 and 578. Thus, two regions near positions 444 and 578 located on the 3 -fold proximal peak are potentially responsible for rAAV5 binding to $\alpha 2,3$-linked sialic acid on host cells. Further studies are required to elucidate the receptor binding phenotype of rAAV5.

Cytotoxicity of rAAV5-RGD1 and-TnC4-mediated sc39TK expression in conjunction with GCV treatment suggests that genetic modification of rAAV5 at the capsid provides a reasonable basis for transductional selectivity and specificity of these mutant viruses for cancer cells. However, our data show that the TEs of re-targeted rAAV5 vectors were not as high as expected, which limits their practical utility. To overcome this limitation, we further investigated the effectiveness of combination treatment with chemical compounds, including anti-cancer agents or protease inhibitors $(33,34)$. Several HDAC inhibitors significantly enhance adenovirus-mediated and rAAV2-mediated transduction efficiencies (34). SAHA, a HDAC inhibitor, was approved by the US Food and Drug Administration for treatment of cutaneous T-cell lymphoma in
2006 (35). As expected, SAHA clearly improved re-targeted rAAV5-mediated gene transfer in a dose-dependent manner. HDAC inhibitors may enhance the utility of re-targeted rAAV5-mediated therapeutic antitumoral gene transduction strategies, promoting synergistic therapeutic effects.

The transduction potencies of genetically modified rAAV5s varied significantly in different mutants. The TE of rAAV5-RGD1 was the most superior, followed by rAAV5TnC3 and -TnC4 (data not shown). In contrast, mutants harboring the sLeX-binding motif did not show any specific transducing ability in sLeX-positive cancer cells (data not shown). Discrepancies in the TEs of rAAV5 mutants may primarily be attributed to differences in binding affinity to their receptors. Trafficking steps, such as inefficient endocytic processes or premature virus degradation by proteasomes following virus entry, may be another reason for variations in the TEs of mutant viruses. Recent studies have shown that the mutation of a surface-exposed tyrosine of rAAV2, 8 and 9 enhances transduction, both in vivo and in vitro, through the evasion of tyrosine phosphorylation and prevention of proteasome-mediated degradation (36). Accordingly, similar types of tyrosine mutations in rAAV5 may increase the TE by allowing mutant rAAV5 to escape from the ubiquitin-proteasome system. We are currently investigating this possibility by generating various mutations at the possible ubiquitin recognition sites.

\section{Acknowledgements}

This study was partly supported by grants to H. Lee from the Korea Science and Engineering Foundation (M1053404002) and the National Research Foundation of Korea (NRF-2010-R13-0029521), Republic of Korea.

\section{References}

1. Hacker UT, Wingenfeld L, Kofler DM, Schuhmann NK, Lutz S, Herold T, King SB, Gerner FM, Perabo L, Rabinowitz J, McCarty DM, Samulski RJ, Hallek M and Buning H: Adenoassociated virus serotypes 1 to 5 mediated tumor cell directed gene transfer and improvement of transduction efficiency. J Gene Med 7: 1429-1438, 2005.

2. Li C, Bowles DE, van Dyke T and Samulski RJ: Adenoassociated virus vectors: potential applications for cancer gene therapy. Cancer Gene Ther 12: 913-925, 2005.

3. Daya S and Berns KI: Gene therapy using adeno-associated virus vectors. Clin Microbiol Rev 21: 583-593, 2008

4. Higano CS, Corman JM, Smith DC, Centeno AS, Steidle CP, Gittleman M, Simons JW, Sacks N, Aimi J and Small EJ: Phase $1 / 2$ dose-escalation study of a GM-CSF-secreting, allogeneic, cellular immunotherapy for metastatic hormone-refractory prostate cancer. Cancer 113: 975-984, 2008.

5. Mueller $\mathrm{C}$ and Flotte TR: Clinical gene therapy using recombinant adeno-associated virus vectors. Gene Ther 15: 858-863, 2008.

6. Lee HS, Shin OK, Kim SJ, Lee WI, Jeong S, Park K, Choe H and Lee H: Efficient gene expression by self-complementary adenoassociated virus serotype 2 and 5 in various human cancer cells. Oncol Rep 18: 611-616, 2007.

7. Thorsen F, Afione S, Huszthy PC, Tysnes BB, Svendsen A, Bjerkvig R, Kotin RM, Lonning PE and Hoover F: Adenoassociated virus (AAV) serotypes 2, 4 and 5 display similar transduction profiles and penetrate solid tumor tissue in models of human glioma. J Gene Med 8: 1131-1140, 2006.

8. Blacklow NR, Hoggan MD, Sereno MS, Brandt CD, Kim HW, Parrott RH and Chanock RM: A seroepidemiologic study of adenovirus-associated virus infection in infants and children. Am J Epidemiol 94: 359-366, 1971. 
9. Halbert CL, Miller AD, McNamara S, Emerson J, Gibson RL, Ramsey B and Aitken ML: Prevalence of neutralizing antibodies against adeno-associated virus (AAV) types 2, 5, and 6 in cystic fibrosis and normal populations: Implications for gene therapy using AAV vectors. Hum Gene Ther 17: 440-447, 2006

10. Gao G, Vandenberghe LH, Alvira MR, Lu Y, Calcedo R, Zhou X and Wilson JM: Clades of adeno-associated viruses are widely disseminated in human tissues. J Virol 78: 6381-6388, 2004.

11. Nathwani AC, Gray JT, McIntosh J, Ng CY, Zhou J, Spence Y, Cochrane M, Gray E, Tuddenham EG and Davidoff AM: Safe and efficient transduction of the liver after peripheral vein infusion of self-complementary AAV vector results in stable therapeutic expression of human FIX in nonhuman primates. Blood 109: 1414-1421, 2007.

12. K.I. Berns CRP: Parvoviridae. In: Field's Virology. D.M. Knipe PMH (ed). Lippincott Williams \& Wilkins, Philadelphia, 2007.

13. Cruet-Hennequart S, Maubant S, Luis J, Gauduchon P, Staedel C and Dedhar S: $\alpha(v)$ integrins regulate cell proliferation through integrin-linked kinase (ILK) in ovarian cancer cells Oncogene 22: 1688-1702, 2003.

14. Moschos SJ, Drogowski LM, Reppert SL and Kirkwood JM: Integrins and cancer. Oncology (Williston Park) 21: 13-20, 2007

15. Wiksten JP, Lundin J, Nordling S, Lundin M, Kokkola A, von Boguslawski $\mathrm{K}$ and Haglund $\mathrm{C}$ : Tenascin-C expression correlates with prognosis in gastric cancer. Oncology 64: 245-250, 2003.

16. Yamaguchi A, Ding K, Maehara M, Goi T and Nakagawara G: Expression of $\mathrm{nm} 23-\mathrm{H} 1$ gene and Sialyl Lewis X antigen in breast cancer. Oncology 55: 357-362, 1998.

17. Girod A, Ried M, Wobus C, Lahm H, Leike K, Kleinschmidt J, Deleage $\mathrm{G}$ and Hallek M: Genetic capsid modifications allow efficient re-targeting of adeno-associated virus type 2. Nat Med 5: 1052-1056, 1999.

18. Grifman M, Trepel M, Speece P, Gilbert LB, Arap W,Pasqualini R and Weitzman MD: Incorporation of tumor-targeting peptides into recombinant adeno-associated virus capsids. Mol Ther 3 : 964-975, 2001.

19. White AF, Mazur M, Sorscher EJ, Zinn KR and Ponnazhagan S: Genetic modification of adeno-associated viral vector type 2 capsid enhances gene transfer efficiency in polarized human airway epithelial cells. Hum Gene Ther 19: 1407-1414, 2008.

20. Work LM, Buning H, Hunt E, Nicklin SA, Denby L, Britton N, Leike K, Odenthal M, Drebber U, Hallek M and Baker AH: Vascular bed-targeted in vivo gene delivery using tropismmodified adeno-associated viruses. Mol Ther 13: 683-693, 2006

21. Xie Q, Bu W, Bhatia S, Hare J, Somasundaram T, Azzi A and Chapman MS: The atomic structure of adeno-associated virus (AAV-2), a vector for human gene therapy. Proc Natl Acad Sci USA 99: 10405-10410, 2002.

22. Sali A and Blundell TL: Comparative protein modelling by satisfaction of spatial restraints. J Mol Biol 234: 779-815, 1993.

23. Brunger AT, Adams PD, Clore GM, DeLano WL, Gros P, Grosse-Kunstleve RW, Jiang JS, Kuszewski J, Nilges M, Pannu NS, Read RJ, Rice LM, Simonson T and Warren GL: Crystallography \& NMR system: A new software suite for macromolecular structure determination. Acta Crystallogr D Biol Crystallogr 54: 905-921, 1998.
24. Chang JW, Lee H, Kim E, Lee Y, Chung SS and Kim JH: Combined antitumor effects of an adenoviral cytosine deaminase/ thymidine kinase fusion gene in rat C6 glioma. Neurosurgery 47: 931-938; discussion 938-939, 2000

25. Wu P, Xiao W, Conlon T, Hughes J, Agbandje-McKenna M, Ferkol T, Flotte T and Muzyczka N: Mutational analysis of the adeno-associated virus type 2 (AAV2) capsid gene and construction of AAV2 vectors with altered tropism. J Virol 74: 8635-8647, 2000

26. Black ME, Newcomb TG, Wilson HM and Loeb LA: Creation of drug-specific herpes simplex virus type 1 thymidine kinase mutants for gene therapy. Proc Natl Acad Sci USA 93: 3525-3529, 1996.

27. Blumenthal M, Skelton D, Pepper KA, Jahn T, Methangkool E and Kohn DB: Effective suicide gene therapy for leukemia in a model of insertional oncogenesis in mice. Mol Ther 15: 183-192, 2007.

28. Grimm D, Kay MA and Kleinschmidt JA: Helper virus-free, optically controllable, and two-plasmid-based production of adeno-associated virus vectors of serotypes 1 to 6 . Mol Ther 7: 839-850, 2003

29. Arnold GS, Sasser AK, Stachler MD and Bartlett JS: Metabolic biotinylation provides a unique platform for the purification and targeting of multiple AAV vector serotypes. Mol Ther 14: 97-106, 2006.

30. Stachler MD and Bartlett JS: Mosaic vectors comprised of modified AAV1 capsid proteins for efficient vector purification and targeting to vascular endothelial cells. Gene Ther 13: 926-931, 2006

31. White K, Buning H, Kritz A, Janicki H, McVey J, Perabo L, Murphy G, Odenthal M, Work LM, Hallek M, Nicklin SA and Baker AH: Engineering adeno-associated virus 2 vectors for targeted gene delivery to atherosclerotic lesions. Gene Ther 15: 443-451, 2008

32. Walters RW, Agbandje-McKenna M, Bowman VD, MoningerTO, Olson NH, Seiler M, Chiorini JA, Baker TS and Zabner J: Structure of adeno-associated virus serotype 5. J Virol 78: 3361-3371, 2004

33. Kim SJ, Nam YR, Shin O, Choi J, Lee B, Chang JW, Kwon YK, Park K and Lee H: Treatment with hydroxyurea and tyrphostin-1 significantly improves the transduction efficiency of recombinant adeno-associated viruses in human cancer cells. Oncol Rep 14: 1475-1479, 2005

34. Okada T, Uchibori R, Iwata-Okada M, Takahashi M, Nomoto T, Nonaka-Sarukawa M, Ito T, Liu Y, Mizukami H, Kume A, Kobayashi E and Ozawa K: A histone deacetylase inhibitor enhances recombinant adeno-associated virus-mediated gene expression in tumor cells. Mol Ther 13: 738-746, 2006.

35. Marks PA and Breslow R: Dimethyl sulfoxide to vorinostat: development of this histone deacetylase inhibitor as an anticancer drug. Nat Biotechnol 25: 84-90, 2007.

36. Petrs-Silva H, Dinculescu A, Li Q, Min SH, Chiodo V, Pang JJ, Zhong L, Zolotukhin S, Srivastava A, Lewin AS and Hauswirth WW: High-efficiency transduction of the mouse retina by tyrosine-mutant AAV serotype vectors. Mol Ther 17: 463-471, 2009 